\title{
Optimal Control of Switched Systems based on Bezier Control Points
}

\author{
FatemeGhomanjani \\ Dept. of Applied Mathematics, Ferdowsi University of Mashhad, Mashhad, Iran \\ fatemeghomanjani@gmail.com \\ Mohammad HadiFarahi \\ Dept. of Applied Mathematics, Ferdowsi University of Mashhad, Mashhad, Iran \\ farahi@um.ac.ir
}

\begin{abstract}
This paper presents a new approach for solving optimal control problems for switched systems. We focus on problems in which a pre-specified sequence of active subsystems is given. For such problems, we need to seek both the optimal switching instants and the optimal continuous inputs. A Bezier control points method is applied for solving an optimal control problem which is supervised by a switched dynamic system. Two steps of approximation exist here. First, the time interval is divided into $k$ sub-intervals. Second, the trajectory and control functions are approximatedby Bezier curves in each subinterval. Bezier curves have been considered as piecewise polynomials of degree $n$, then they will be determined by $n+1$ control points on any subinterval. The optimal control problem is there by converted into a nonlinear programming problem (NLP), which can be solved by known algorithms. However in this paper the MATLAB optimization routine FMINCON is used for solving resulting NLP.
\end{abstract}

Index Terms - switched dynamical system, Bezier control points, optimal control.

\section{Introduction}

A switched system is composed of some subsystems and a switching law. The switching law defines the order of the subsystems which have to be activated at specified switching instants during the planning horizon. Many real world applications face switched systems, such as the control of mechanical systems, automotive industry, aircraft and air traffic control and switching power convertors (details can be found in [1-7]).

The objective of problems related to optimal control of switched systems is to find a switching law and a control function in a way that some performance criteria are minimized with respect to imposed constraints on state and control variables. These groups of optimal control problems are of theoretical and practical importance, so they have been taken into special consideration ([8-12]).
For switched system with linear component systems, the defect of optimal control switching has absorbed several researcher's attention recently. For instance, Egerstedt, et al. [13] presented such an accurate method and algorithm that is able to compute the minimum number of switching for changing from one state to another and $\mathrm{Xu}$, and Antsakalis [14], addressed the problem of determining the switch instants.

These results, like most of the results in the literature, make open-loop and are based on pre-selecting a finite number of switching and optimization of the switching instance against a cost functional. For these open-loop solutions, the result of optimization can be enhanced if the number of switching is allowed to increase but since these methods use a search algorithm, the cast of computation increases very suddenly. $\mathrm{Xu}$, and Zhai [15] continued their recent study on practical stabilizability of discrete-time (DT) switched systems, and they proved a sufficient condition for $\varepsilon$-practical asymptotic stabilizability. On the basis of their approach, they also presented several new sufficient conditions for global $\varepsilon$ practical asymptotic stabilizability of such a class of systems.

Niua, and Zhaoa [16] surveyed the problem of stabilization and $L_{2}$-gain analysis for a class of cascade switched nonlinear systems by using the average dwelltime method. First, when all subsystems were stabilizable, they designed a state feedback controller and an average dwell-time scheme, which guaranteed that the corresponding closed-loop system was globally asymptotically stable and had a weighted $L_{2}$-gain. Then, they extended the result to the case where not all subsystems were stabilizable, under the condition that the activation time ratio between stabilizable subsystems and unstabilizable ones was not less than a specified constant, also they derived sufficient conditions for the stabilization and weighted $L_{2}$-gain property.

In this work, a class of optimal switching problems have been considered such that the switching instants and the control function have to be found optimally 
under the condition of defined order of the subsystems. The purpose of this paper is to propose an efficient computational algorithm based on Bezier curves introduced in [3] for solving these type of optimal switching problems.

This paper is organized as follows: In Sections 2 and 3 , the basic problem will be introduced. Two examples will be presented in Section 4 and solved by the proposed method. And at last, Section 5 will discuss about conclusion.

\section{Switched Systems}

A switched systems composing of following subsystems considered as follows:

$$
\dot{\boldsymbol{x}}(t)=F_{i}(t, \boldsymbol{x}(t), \boldsymbol{u}(t)), i \in I=\{1,2, \ldots, N\},
$$

where $\boldsymbol{x}(t) \in R^{p}, \boldsymbol{u}(t) \in R^{m}$ and for each $i \in$ $I, F_{i}: R^{p+m+1} \rightarrow R^{p}$ is continuously differentiable with respect to its arguments. To control a switched system, a continuous input as well as a switching sequence have to been selected. A switching sequence with $t \in$ $\left[t_{0}, t_{f}\right]$ adjusts the order of active subsystems and is defined as:

$$
\sigma=\left\{\left(T_{0}, i_{0}\right),\left(T_{1}, i_{1}\right), \ldots,\left(T_{k}, i_{K}\right)\right\},
$$

where $0 \leq K<\infty, t_{0}=T_{0} \leq T_{1} \leq \cdots \leq T_{K} \leq t_{f}$, and $i_{k} \in I$ for $k=0,1, \ldots, K$ Here, $\left(T_{k}, i_{k}\right)$ indicates that at instant $T_{k}$, the system switches from subsystem $i_{k-1}$ to subsystem $i_{k}$; therefore subsystem $i_{k}$ is active during time interval $\left[T_{k}, T_{k+1}\right)\left(\left[T_{k}, T_{k+1}\right]\right.$ if $\left.k=K\right)$. In order a switched system to be well-behaved, only nonZeno sequences are considered which switch at most a finite number of times in $\left[t_{0}, t_{f}\right]$, though different sequences may be of different numbers of switchings. If $\sigma$ is regarded as a discrete input, then the overall control input on the system is the pair $(\sigma, u)$. It is mentioned that the discriminately factor for a switched system from a general hybrid system is its continuous state which doesn't represent jumps at switching instants. Hence, the computation of continuous inputs becomes favorable via the application of conventional optimal control methods.

This paper goals at minimizing cost functional over two subsystems solution as the following form

$$
\begin{gathered}
\min I=\psi\left(\boldsymbol{x}\left(t_{f}\right)\right)+\int_{t_{0}}^{t_{f}} L(\boldsymbol{x}(t), \boldsymbol{u}(t)) d t \\
\text { s.t. } \dot{\boldsymbol{x}}(t)=\boldsymbol{F}(\boldsymbol{x}(t), \boldsymbol{u}(t)), \quad t \in\left[t_{0}, T_{1}\right), \\
\dot{\boldsymbol{x}}(t)=\boldsymbol{G}(\boldsymbol{x}(t), \boldsymbol{u}(t)), \quad t \in\left[T_{1}, t_{f}\right] \\
\boldsymbol{x}(t)=\boldsymbol{\phi}(t), t \leq t_{0}
\end{gathered}
$$

where $\quad \boldsymbol{x}(t)=\left(x_{1}(t) \ldots x_{p}(t)\right)^{T} \in R^{p}, \boldsymbol{u}(t)=$ $\left(u_{1}(t) \ldots u_{m}(t)\right)^{T} \in R^{m} \quad, \quad$ and $\quad \boldsymbol{\phi}(t)=$ $\left(\phi_{1}(t) \ldots \phi_{p}(t)\right)^{T}$ are known vectors functions, and $t_{0}$ and $t_{f}$ are known values in $R$. We assume $\boldsymbol{F}(t)=$ $\left(f_{1}(.) \ldots f_{p}(.)\right)^{T}$ and $\boldsymbol{G}(t)=\left(g_{1}(.) \ldots g_{p}(.)\right)^{T}$ are vector functions, which their elements assumed to be polynomials defined on $\left[t_{0}, T_{1}\right)$ and $\left[T_{1}, t_{f}\right]$, respectively. We need to impose continuity on $\boldsymbol{x}(t)$ and its first derivative which these constraints is appeared in Section 3 .

Without lose of generality just for simplicity, the case of two subsystems are considered in which the subsystems (a) and (b) in (3) are active in $t \in$ $\left[t_{0}, T_{1}\right)$ and $t \in\left[T_{1}, t_{f}\right]$, respectively ( $T_{1}$ is switching instant which has to be determined). Applying developed methods on problems with several subsystems with more than one switching is straightforward [14].

Problem (3) can be converted into a new problem. A state variable $y$ is introduced corresponding to switching instant $T_{1}$. Let $y$ satisfy

$$
\begin{gathered}
\frac{d y}{d t}=0, \\
y(0)=T_{1} .
\end{gathered}
$$

Now, a new variable $\xi$ is introduced. Define a piecewise linear relationship between $t$ and $\xi$ as follows:

$$
t=\left\{\begin{array}{c}
t_{0}+\left(y-t_{0}\right) \xi, 0 \leq \xi \leq 1 \\
y+\left(t_{f}-y\right)(\xi-1), 1 \leq \xi \leq 2
\end{array}\right.
$$

Obviously, $\xi=0$ corresponds to $t=t_{0}, \xi=1$ to $t=T_{1}$, and $\xi=2$ to $t=t_{f}$. By substituting $y$ and $\xi$ in $\boldsymbol{F}($.$) and \boldsymbol{G}($.$) in equations (a) and (b) of (3), now$ these equations are converted into the following equivalent forms:

$$
\begin{aligned}
\frac{d x(\xi)}{d \xi} & =\left(y-t_{0}\right) \boldsymbol{F}_{1}(\boldsymbol{x}(\xi), \boldsymbol{u}(\xi)), \\
\frac{d y}{d \xi} & =0,
\end{aligned}
$$

where $\xi \in[0,1)$ and

$$
\begin{aligned}
& \frac{d x(\xi)}{d \xi}=\left(t_{f}-y\right) \boldsymbol{G}_{1}(\boldsymbol{x}(\xi), \boldsymbol{u}(\xi)), \\
& \frac{d y}{d \xi}=0,
\end{aligned}
$$

where $\xi \in[1,2]$, and

$$
\boldsymbol{x}(\xi)=\boldsymbol{\phi}\left(t_{0}+\left(y-t_{0}\right) \xi\right), \xi \leq 0 .
$$

Now the target is to find $y$ and $\boldsymbol{u}(\xi), \xi \in[0,2]$ such that the cost functional

$$
\begin{gathered}
I=\psi(\boldsymbol{x}(2))+\int_{0}^{1}\left(y-t_{0}\right) L(\boldsymbol{x}, \boldsymbol{u}) d \xi+\int_{1}^{2}\left(t_{f}-\right. \\
y) L((\boldsymbol{x}, \boldsymbol{u}) d \xi,
\end{gathered}
$$

to be minimized.

Remark 1: The equivalent problem (7)-(12) provides us with no more varying switching instant and consequently it is conventional. Since $y$ actually is an 
unknown constant while $\xi \in[0,2]$, thus $y$ isregarded as unknown parameter for optimal control problem with cost (12) and subsystems (7)-(11), that is, Problem (7)(11) with cost functional (12) can be regarded as an optimal control problem parameterized by switching instant $y$. It is worth noting that by considering $y$ as a parameter, thedimension of new problem is the same as that of first problem. In fact, this situation is valid when considering more than one switching.

\section{Statement of Problem}

Consider the optimal control of time varying system (7)-(11) with cost functional (12). Divide the interval $[0,2]$ into a set of grid points such that

$$
\xi_{i}=0+i h, i=0,1, \ldots, k,
$$

where $h=\frac{2}{k}$, and $k$ is a positive integer. Let $S_{i}=$ $\left[\xi_{j-1}, \xi_{j}\right]$ for $j=1,2, \ldots, k$. Then, for $\xi \in S_{j}$ the above optimal control problem can be decomposed to the following suboptimal control problems:

$$
\begin{aligned}
& \min I_{j}=C_{j}+I_{\text {first }}+I_{\text {second }} \\
& \text { s.t. } I_{\text {first }}=\int_{\xi_{j-1}}^{\xi_{j}} \chi_{[0,1)}\left(y-t_{0}\right) L\left(\boldsymbol{x}_{j}, \boldsymbol{u}_{j}\right) d \xi, \\
& I_{\text {second }}=\int_{\xi_{j-1}}^{\xi_{j}} \chi_{[1,2]}\left(t_{f}-y\right) L\left(\boldsymbol{x}_{j}, \boldsymbol{u}_{j}\right) d \xi, \quad \xi \in[1,2], \\
& \frac{d \boldsymbol{x}_{\boldsymbol{j}}(\xi)}{d \xi}=\chi_{[0,1)}\left(y-t_{0}\right) \boldsymbol{F}_{1}(\boldsymbol{x}(\xi), \boldsymbol{u}(\xi)), \xi \in S_{j}, \\
& \frac{d \boldsymbol{x}_{\boldsymbol{j}}(\xi)}{d \xi}=\chi_{[1,2]}\left(t_{f}-y\right) \boldsymbol{G}_{1}(\boldsymbol{x}(\xi), \boldsymbol{u}(\xi)), \xi \in S_{j}, \\
& \frac{d y}{d \xi}=0, \xi \in[1,2], \\
& \boldsymbol{x}_{j}(\xi)=\boldsymbol{\phi}\left(t_{0}+\left(y-t_{0}\right) \xi\right), \xi \leq 0,
\end{aligned}
$$

where $\quad \boldsymbol{x}_{j}(\xi)=\left(x_{1}^{j}(\xi) \ldots x_{p}^{j}(\xi)\right)^{T}, \quad$ and $\quad \boldsymbol{u}_{j}(\xi)=$ $\left(u_{1}^{j}(\xi) \ldots u_{m}^{j}(\xi)\right)^{T}$ are respectively vectors of $\boldsymbol{x}(\xi)$ and $\boldsymbol{u}(\xi)$ which are considered in $\xi \in S_{j}$, and $\chi_{[0,1)}$ and $\chi_{[1,2]}$ are respectively characteristic functions of $\boldsymbol{F}_{1}(\boldsymbol{x}(\xi), \boldsymbol{u}(\xi))$ and $\boldsymbol{G}_{1}(\boldsymbol{x}(\xi), \boldsymbol{u}(\xi))$. Also

$$
C_{j}=\left\{\begin{array}{rr}
\psi(x(2)), & j=k \\
0, & j \neq k
\end{array}\right.
$$

Zheng, et al. [3] shown the convergence of the subdivision scheme as the interval width approaches zero. Our strategy is using Bezier curves to approximate the solutions $\boldsymbol{x}_{j}(\xi)$ and $\boldsymbol{u}_{j}(\xi)$ by $\boldsymbol{v}_{j}(\xi)$ and $\boldsymbol{w}_{j}(\xi)$ respectively, where $\boldsymbol{v}_{j}(\xi)$ and $\boldsymbol{w}_{j}(\xi)$ are given below. Individual Bezier curves that are defined over the subintervals are joined together to form the Bezier spline curves. For $j=1,2, \ldots, k$, define the Bezier polynomials of degree $\mathrm{n}$ that approximate the actions of $\boldsymbol{x}_{j}(\xi)$ and $\boldsymbol{u}_{j}(\xi)$ over the interval $\left[\xi_{j-1}, \xi_{j}\right]$ as follows

$$
\begin{gathered}
\boldsymbol{v}_{j}(\xi)=\sum_{r=0}^{n} \boldsymbol{a}_{r}^{j} B_{r, n}\left(\frac{\xi-\xi_{j-1}}{h}\right), \\
\boldsymbol{w}_{j}(\xi)=\sum_{r=0}^{n} \boldsymbol{b}_{r}^{j} B_{r, n}\left(\frac{\xi-\xi_{j-1}}{h}\right),
\end{gathered}
$$

where

$$
B_{r, n}\left(\frac{\xi-\xi_{j-1}}{h}\right)=\left(\begin{array}{l}
r \\
n
\end{array}\right) \frac{1}{h^{n}}\left(\xi_{j}-\xi\right)^{n-r}\left(\xi-\xi_{j-1}\right)^{r},
$$

is the Bernstein polynomial of degree $\mathrm{n}$ over the interval $\left[\xi_{j-1}, \xi_{j}\right], \boldsymbol{a}_{r}^{j}$ and $\boldsymbol{b}_{r}^{j}$ are respectively $p$ and $m$ ordered vectors from the control points (see [3]). By substituting (14) in (13), one may define $R_{1, j}(\xi)$ for $\xi \in\left[\xi_{j-1}, \xi_{j}\right]$ as

$$
\begin{aligned}
R_{1, j}(\xi)=\chi_{[0,1)}\left(y-t_{0}\right) L\left(\boldsymbol{x}_{j}, \boldsymbol{u}_{j}\right) \\
\\
+\chi_{[1,2]}\left(t_{f}-y\right) L\left(\boldsymbol{x}_{j}, \boldsymbol{u}_{j}\right), j \\
=1,2, \ldots, k .
\end{aligned}
$$

Let $\quad \boldsymbol{v}(\xi)=\sum_{j=1}^{k} \chi_{j}^{1}(\xi) \boldsymbol{v}_{j}(\xi) \quad$ and $\quad \boldsymbol{w}(\xi)=$ $\sum_{j=1}^{k} \chi_{j}^{2}(\xi) \boldsymbol{w}_{j}(\xi)$ where $\chi_{j}^{1}(\xi)$ and $\chi_{j}^{2}(\xi)$ are respectively characteristic functions of $\boldsymbol{v}_{j}(\xi)$ and $\boldsymbol{w}_{j}(\xi)$ for $\xi \in$ $\left[\xi_{j-1}, \xi_{j}\right]$. Beside the boundary conditions on $\boldsymbol{v}(\xi)$, in nodes, there are also continuity constraints imposed on each successive pair of Bezier curves. Since the differential equation is of first order, the continuity of $\boldsymbol{x}$ (or $\boldsymbol{v}$ ) and its first derivative gives

$$
\boldsymbol{v}_{j}^{(s)}\left(\xi_{j}\right)=\boldsymbol{v}_{j+1}^{(s)}\left(\xi_{j}\right), s=0,1, j=1,2, \ldots, k-1 .
$$

Thus the vector of control points $\boldsymbol{a}_{r}^{j}(r=0,1, n-$ $1, n)$ must satisfy

$$
\begin{aligned}
& \boldsymbol{a}_{n}^{j}\left(\xi_{j}-\xi_{j-1}\right)^{n}=\boldsymbol{a}_{0}^{j+1}\left(\xi_{j+1}-\xi_{j}\right)^{n}, \\
& \quad\left(\boldsymbol{a}_{n}^{j}-\boldsymbol{a}_{n-1}^{j}\right)\left(\xi_{j}-\xi_{j-1}\right)^{n-1}=\left(\boldsymbol{a}_{1}^{j+1}-\boldsymbol{a}_{0}^{j+1}\right)\left(\xi_{j+1}-\right. \\
& \left.\xi_{j}\right)^{n-1} .
\end{aligned}
$$

One may recall that $\boldsymbol{a}_{r}^{j}$ is an $p$ ordered vector. This approach is called the subdivision scheme (or $h$ refinement in the finite element literature).

Note 1: If we consider the $C^{1}$ continuity of $\boldsymbol{w}$, the following constraints will be added to constraints (16),

$$
\begin{gathered}
\boldsymbol{b}_{n}^{j}\left(\xi_{j}-\xi_{j-1}\right)^{n}=\boldsymbol{b}_{0}^{j+1}\left(\xi_{j+1}-\xi_{j}\right)^{n}, \\
\left(\boldsymbol{b}_{n}^{j}-\boldsymbol{b}_{n-1}^{j}\right)\left(\xi_{j}-\xi_{j-1}\right)^{n-1} \\
=\left(\boldsymbol{b}_{1}^{j+1}-\boldsymbol{b}_{0}^{j+1}\right)\left(\xi_{j+1}-\xi_{j}\right)^{n-1},
\end{gathered}
$$

where the so-called $\boldsymbol{b}_{r}^{j}(r=0,1, n-1, n)$ is an $m$ ordered vector.

Now, we define residual function in $S=\bigcup_{j=1}^{k} S_{j}$ as follows 


$$
R=\sum_{j=1}^{k}\left\|C_{j}\right\|^{2}+\sum_{j=1}^{k}\left\|R_{1, j}\right\|^{2} d \xi,
$$

where $\|$.$\| is L_{2}$ norm and $M$ is a sufficiently large penalty parameter. Our aim is to solve the following problem over $S=\bigcup_{j=1}^{k} S_{j}$ :

$\min R$

$$
\begin{aligned}
& \text { s.t. } \frac{d \boldsymbol{v}_{\boldsymbol{j}}(\xi)}{d \xi}=\chi_{[0,1)}\left(y-t_{0}\right) \boldsymbol{F}_{1}(\boldsymbol{v}(\xi), \boldsymbol{w}(\xi)), \\
& \xi \in S_{j} \text {, } \\
& \frac{d \boldsymbol{v}_{\boldsymbol{j}}(\xi)}{d \xi}=\chi_{[1,2]}\left(t_{f}-y\right) \boldsymbol{G}_{1}(\boldsymbol{v}(\xi), \boldsymbol{w}(\xi)), \xi \in S_{j}, \\
& \boldsymbol{v}_{j}^{(s)}\left(\xi_{j}\right)=\boldsymbol{v}_{j+1}^{(s)}\left(\xi_{j}\right), s=0,1, j=1,2, \ldots, k-1 \\
& \boldsymbol{v}_{j}(\xi)=\boldsymbol{\phi}\left(t_{0}+\left(y-t_{0}\right) \xi\right), \xi \leq 0,
\end{aligned}
$$

The optimal control problem is thereby converted into a nonlinear programming problem (NLP), which can be solved by known algorithms. However in this paper the MATLAB optimization routine FMINCON is used for solving resulting NLP.

Note 2: In problem (3), if $\boldsymbol{x}\left(t_{f}\right)$ is unknown, then we set $C_{k}=0$.

\section{Numerical Examples}

In applying the method, in Example 4.1 and 4.2, we choose the Bezier curves as piecewise polynomials of degree 3 .

Example IV.1: Consider the following optimal control problem (see[17]):

$$
\begin{gathered}
\min I=\frac{1}{2}\left(\left(x_{1}(9)-2.4428\right)^{2}+\left(x_{2}(9)-2\right)^{2}\right) \\
+\int_{0}^{9} u^{2}(t) d t
\end{gathered}
$$

$$
\begin{aligned}
& \text { s.t. } \dot{x}(t)=\left[\begin{array}{cc}
-1.2 & 0 \\
0 & -1
\end{array}\right] \boldsymbol{x}(t)+\left[\begin{array}{l}
1 \\
0
\end{array}\right] u(t), 0 \leq t \leq T_{1} \text {, } \\
& \dot{\boldsymbol{x}}(t)=\left[\begin{array}{cc}
1 & 0 \\
0 & 0.8
\end{array}\right] \boldsymbol{x}(t)+\left[\begin{array}{l}
0 \\
1
\end{array}\right\rfloor u(t), T_{1} \leq t \leq 9, \\
& \boldsymbol{x}(0)=\left\lfloor\begin{array}{ll}
x_{1}(0) & x_{2}(0)
\end{array}\right]^{T}=\left[\begin{array}{ll}
2 & 2
\end{array}\right]^{T} .
\end{aligned}
$$

Let $k=9$ and, $n=3$. By using mentioned algorithm with active-set method, after 2 iterations, we found that the switched instant is $T_{1}=4$, and the approximated objective function by this method is 0.000390688212 . From (17), one can find the following solution.

$$
\begin{gathered}
x_{1}(t)=\left\{\begin{array}{cr}
2-2.392839288 t+1.316792458 t^{2}-0.3205476314 t^{3} & 0 \leq t \leq 1, \\
1.808706695-1.782704037 t+0.6704018706 t^{2}-0.09299899010 t^{3} & 1 \leq t \leq 2, \\
1.391378195-1.131009129 t+0.3317033376 t^{2}-0.03440738807 t^{3} & 2 \leq t \leq 3, \\
0.3004422490-0.03208509009 t-0.03726737298 t^{2}+0.006885212840 t^{3} & 3 \leq t \leq 4, \\
1.478700005-0.9355631149 t+0.1935483102 t^{2}-0.01276160884 t^{3} & 4 \leq t \leq 5, \\
-0.9361084791+0.6205686165 t-0.1391273643 t^{2}+0.01084672467 t^{3} & 5 \leq t \leq 6, \\
-6.680256322+3.458585025 t-0.6064538469 t^{2}+0.03649395970 t^{3} & 6 \leq t \leq 7, \\
-28.20579803+12.54029976 t-1.883339175 t^{2}+0.09632171390 t^{3} & 7 \leq t \leq 8, \\
-103.2191796+40.46646710 t-5.348628750 t^{2}+0.2396470569 t^{3} & 8 \leq t \leq 9,
\end{array}\right. \\
x_{2}(t)=\left\{\begin{array}{cc}
1.90435964-1.676728455 t+0.5877731771 t^{2}-0.07888302320 t^{3} & 1 \leq t \leq 1, \\
1.536528977-1.099341993 t+0.2862597308 t^{2}-0.02649407959 t^{3} & 2 \leq t \leq 3, \\
0.2850772820+0.07889166302 t-0.08207880858 t^{2}+0.01172064559 t^{3} & 3 \leq t \leq 4, \\
2.635246643-1.610926035 t+0.3221732854 t^{2}-0.02045016803 t^{3} & 4 \leq t \leq 5, \\
-3.434516208+1.947584374 t-0.3728593363 t^{2}+0.024777404274 t^{3} & 5 \leq t \leq 6, \\
-3.885208398+2.045722138 t-0.3680142417 t^{2}+0.02332701590 t^{3} & 6 \leq t \leq 7, \\
-12.99767967+5.958480946 t-0.9280389254 t^{2}+0.0500452932 t^{3} & 7 \leq t \leq 8, \\
-43.50724416+17.39883072 t-2.357990534 t^{2}+0.1096227722 t^{3} & 8 \leq t \leq 9,
\end{array}\right.
\end{gathered}
$$

and,

$$
u(t)=\left\{\begin{array}{cc}
-0.1593519913 t+0.4357306624 t^{2}-0.2702418138 t^{3} & 0 \leq t \leq 1, \\
0.4384628419-0.8859669733 t+0.5735721005 t^{2}-0.1199311119 t^{3} & 1 \leq t \leq 2, \\
1.082281030-1.357312035 t+0.5620535216 t^{2}-0.07681283036 t^{3} & 2 \leq t \leq 3, \\
5.535843209-4.849301324 t+1.405525654 t^{2}-0.1349181455 t^{3} & 3 \leq t \leq 4, \\
18.53870467-12.50786952 t+2.796773229 t^{2}-0.2072392371 t^{3} & 4 \leq t \leq 5, \\
11.75230180-6.197185809 t+1.086868089 t^{2}-0.06339433465 t^{3} & 5 \leq t \leq 6, \\
1.142153286-0.5503729595 t+0.08877618174 t^{2}-0.004780538000 t^{3} & 6 \leq t \leq 7, \\
14.61752960-5.924197313 t+0.7991315285 t^{2}-0.03587679992 t^{3} & 7 \leq t \leq 8, \\
48.15080771-17.19006978 t+2.043727235 t^{2}-0.08091668965 t^{3} & 8 \leq t \leq 9 .
\end{array}\right.
$$

The computation takes 1000 seconds of CPU time when it is performed using Matlab 2010a on an AMD Athelon X 4 PC with 2 GB of RAM. The graphs of approximated $x_{1}(t), x_{2}(t)$ and approximated control are shown respectively in Figures 1, 2 and 3. 
Note that the exact optimal solution for this problem is (see [17])

$$
T_{1}^{*}=4, u(t) \equiv 0, \min I=0 .
$$

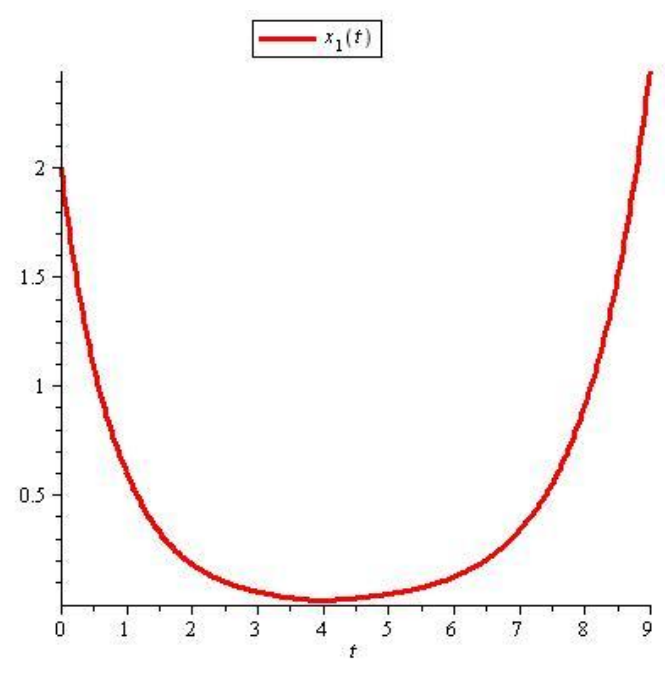

Fig.1 The graph of approximated $x_{1}(t)$

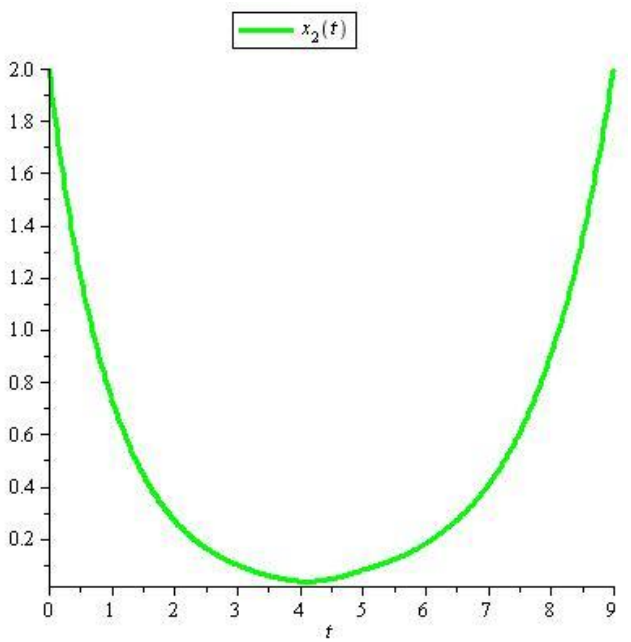

Fig.2 The graph of approximated $x_{2}(t)$

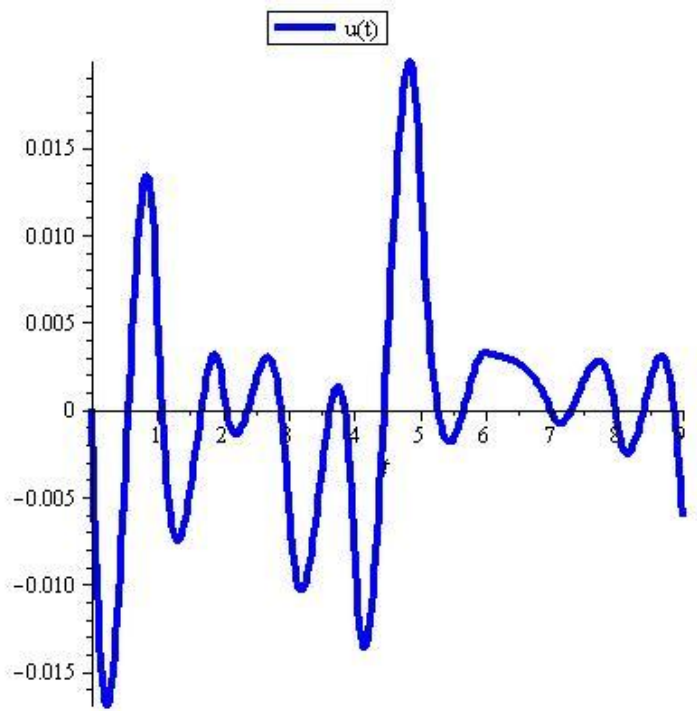

Fig.3 The graph of approximated control

Example IV.2 Consider the following optimal control problem (see[14]):

$$
\begin{aligned}
& \min I=\frac{1}{2}\left(\left(x_{1}(2)-4\right)^{2}+\left(x_{2}(2)-2\right)^{2}\right) \\
& +\frac{1}{2} \int_{0}^{2}\left(\left(x_{2}(t)-2\right)^{2}+u^{2}(t)\right) d t \\
& \text { s.t. } \dot{x}(t)=\left[\begin{array}{cc}
0.6 & 1.2 \\
-0.8 & 3.4
\end{array}\right] \boldsymbol{x}(t)+\left\lfloor\begin{array}{l}
1 \\
1
\end{array}\right\rfloor u(t), 0 \leq t \leq T_{1} \text {, } \\
& \dot{\boldsymbol{x}}(t)=\left[\begin{array}{cc}
4 & 3 \\
-1 & 0
\end{array}\right] \boldsymbol{x}(t)+\left\lfloor\begin{array}{c}
2 \\
-1
\end{array}\right\rfloor u(t), T_{1} \leq t \leq 2 \text {, } \\
& \boldsymbol{x}(0)=\left\lfloor\begin{array}{ll}
x_{1}(0) & x_{2}(0)
\end{array}\right\rfloor^{T}=\left\lfloor\begin{array}{ll}
0 & 2
\end{array}\right\rfloor^{T} .
\end{aligned}
$$

Let $k=20$ and, $n=3$. By using mentioned algorithm, we found that the switched instant is $T_{1}=0.17$, and the approximated objective function by this method is 9.329921749. From (17), one can find the following solution.

$$
x_{1}(t)=\left\{\begin{array}{cc}
-10.92427311 t-88.99616160 t^{2}+825.9280790 t^{3} & 0 \leq t \leq 0.1, \\
1.915913401-68.06241582 t+478.9926727 t^{2}-1056.059394 t^{3} & 0.1 \leq t \leq 0.2, \\
-3.229687448+34.57688896 t-161.4803114 t^{2}+223.5230136 t^{3} & 0.2 \leq t \leq 0.3, \\
1.109324474-18.25900824 t+46.12527240 t^{2}-42.13422000 t^{3} & 0.3 \leq t \leq 0.4, \\
-0.6947052100-2.099877390 t-0.8448252000 t^{2}+2.484420000 t^{3} & 0.4 \leq t \leq 0.5, \\
-0.5021273950-3.867739620 t+3.915690000 t^{2}-1.505784000 t^{3} & 0.5 \leq t \leq 0.6, \\
-0.5161999930-3.694819410 t+3.456561000 t^{2}-1.155752000 t^{3} & 0.6 \leq t \leq 0.7, \\
-0.8579139600-2.237479440 t+1.384858800 t^{2}-0.1740900000 t^{3} & 0.7 \leq t \leq 0.8, \\
-1.069491944-1.448535120 t+0.4042698000 t^{2}+0.2321590000 t^{3} & 0.8 \leq t \leq 0.9, \\
-1.540205516+0.1226180700 t-1.343798100 t^{2}+0.8804590000 t^{3} & 0.9 \leq t \leq 1, \\
-2.227859986+2.183996040 t-3.403590600 t^{2}+1.566528000 t^{3} & 1 \leq t \leq 1.1, \\
-3.388089573+5.347624890 t-6.279040800 t^{2}+2.437702000 t^{3} & 1.1 \leq t \leq 1.2, \\
-5.335317045+10.21424673 t-10.33335330 t^{2}+3.563565000 t^{3} & 1.2 \leq t \leq 1.3, \\
-8.629786951+17.81489547 t-16.17848760 t^{2}+5.061928000 t^{3} & 1.3 \leq t \leq 1.4, \\
-14.17822747+29.70144651 t-24.66676380 t^{2}+7.082442000 t^{3} & 1.4 \leq t \leq 1.5, \\
-23.43852405+48.21801782 t-37.00846345 t^{2}+9.824446100 t^{3} & 1.5 \leq t \leq 1.6, \\
-38.78543661+76.98657422 t-54.98449581 t^{2}+13.56855380 t^{3} & 1.6 \leq t \leq 1.7, \\
-63.72782578+120.9970447 t-80.86976648 t^{2}+18.64346150 t^{3} & 1.7 \leq t \leq 1.8, \\
-104.8969032+189.5942580 t-118.9693762 t^{2}+25.69710160 t^{3} & 1.8 \leq t \leq 1.9, \\
-166.8730805+287.4592285 t-170.4813861 t^{2}+34.73502100 t^{3} & 1.9 \leq t \leq 2,
\end{array}\right.
$$




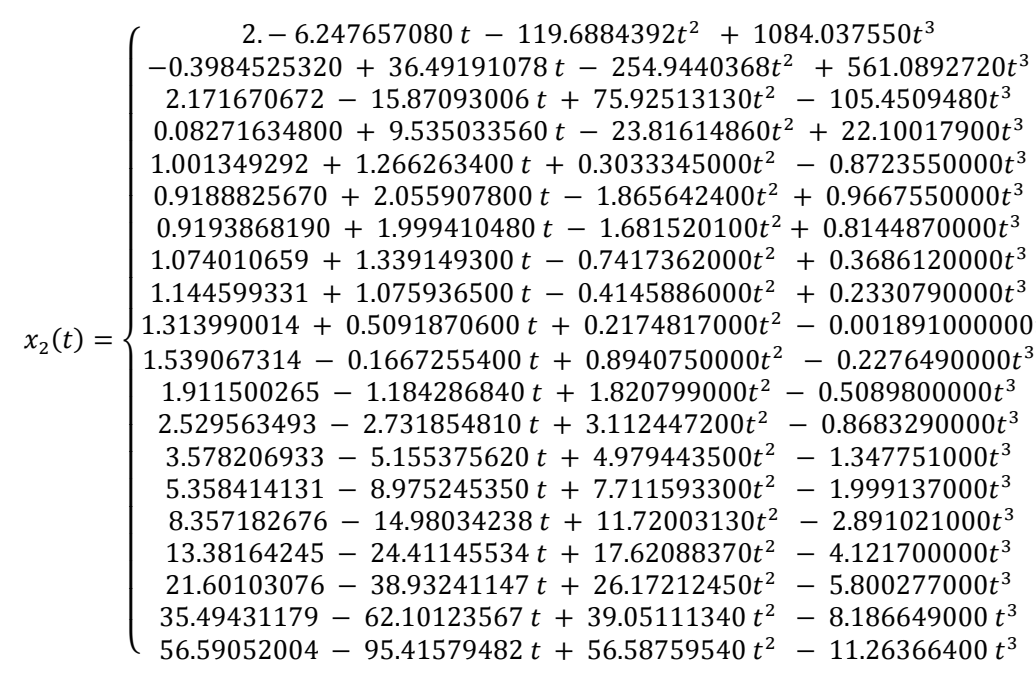

and,
$0 \leq t \leq 0.1$

$0.1 \leq t \leq 0.2$

$0.2 \leq t \leq 0.3$

$0.3 \leq t \leq 0.4$

$0.4 \leq t \leq 0.5$

$0.5 \leq t \leq 0.6$

$0.6 \leq t \leq 0.7$

$0.7 \leq t \leq 0.8$

$0.8 \leq t \leq 0.9$

$0.9 \leq t \leq 1$,

$1 \leq t \leq 1.1$,

$1.1 \leq t \leq 1.2$,

$1.2 \leq t \leq 1.3$

$1.3 \leq t \leq 1.4$,

$1.4 \leq t \leq 1.5$,

$1.5 \leq t \leq 1.6$,

$1.6 \leq t \leq 1.7$,

$1.7 \leq t \leq 1.8$,

$1.8 \leq t \leq 1.9$,

$1.9 \leq t \leq 2$,

$$
u(t)=\left\{\begin{array}{cc}
-12 .-271.9368459 t+4813.469301 t^{2}-12235.54335 t^{3} & 0 \leq t \leq 0.1, \\
-107.3673603+1966.962301 t-11354.30555 t^{2}+20919.65079 t^{3} & 0.1 \leq t \leq 0.2, \\
103.1832786-1187.617197 t+4400.191513 t^{2}-5307.176954 t^{3} & 0.2 \leq t \leq 0.3, \\
-62.01583519+497.3348902 t-1326.185276 t^{2}+1177.541515 t^{3} & 0.3 \leq t \leq 0.4, \\
20.06701776-137.4718584 t+308.7949736 t^{2}-224.9115092 t^{3} & 0.4 \leq t \leq 0.5, \\
-8.365959965+41.83694681 t-67.24451456 t^{2}+37.39606800 t^{3} & 0.5 \leq t \leq 0.6, \\
-0.9632493676+2.764604054 t+1.307372980 t^{2}-2.5946008000 t^{3} & 0.6 \leq t \leq 0.7, \\
-0.7599670345+3.225506185 t-1.254075950 t^{2}-0.4686645000 t^{3} & 0.7 \leq t \leq 0.8, \\
-0.4567610441+2.548305161 t-0.9823514700 t^{2}-0.3423927000 t^{3} & 0.8 \leq t \leq 0.9, \\
1.252018664-2.353197923 t+3.581063870 t^{2}-1.705620900 t^{3} & 0.9 \leq t \leq 1, \\
2.995233768-6.617740527 t+6.880503770 t^{2}-2.483733300 t^{3} & 1 \leq t \leq 1.1, \\
6.141198540-13.87490271 t+12.27543144 t^{2}-3.754168200 t^{3} & 1.1 \leq t \leq 1.2, \\
11.09308293-24.38528583 t+19.47631084 t^{2}-5.321697700 t^{3} & 1.2 \leq t \leq 1.3, \\
19.22373651-40.45418733 t+29.76458490 t^{2}-7.428327100 t^{3} & 1.3 \leq t \leq 1.4, \\
32.45124930-64.88261913 t+44.41615156 t^{2}-10.25076840 t^{3} & 1.4 \leq t \leq 1.5, \\
54.02509013-102.3256824 t+65.57511486 t^{2}-14.10763160 t^{3} & 1.5 \leq t \leq 1.6, \\
88.27499645-158.2371555 t+95.32784724 t^{2}-19.22446370 t^{3} & 1.6 \leq t \leq 1.7, \\
144.8752853-246.1052081 t+139.9474017 t^{2}-26.58760470 t^{3} & 1.7 \leq t \leq 1.8, \\
229.3779020-369.2999893 t+198.5873282 t^{2}-35.63173580 t^{3} & 1.8 \leq t \leq 1.9, \\
364.6997357-559.1462311 t+285.9696600 t^{2}-48.76254090 t^{3} & 1.9 \leq t \leq 2 .
\end{array}\right.
$$

The graphs of approximated trajectories, and approximated control are shown respectively in Figures 4 and 5.

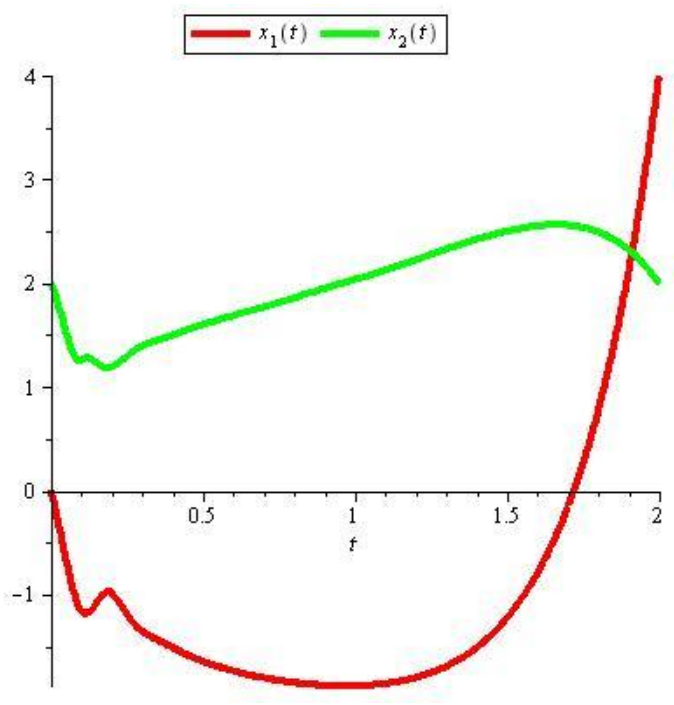

Fig.4 The graphs of approximated trajectories

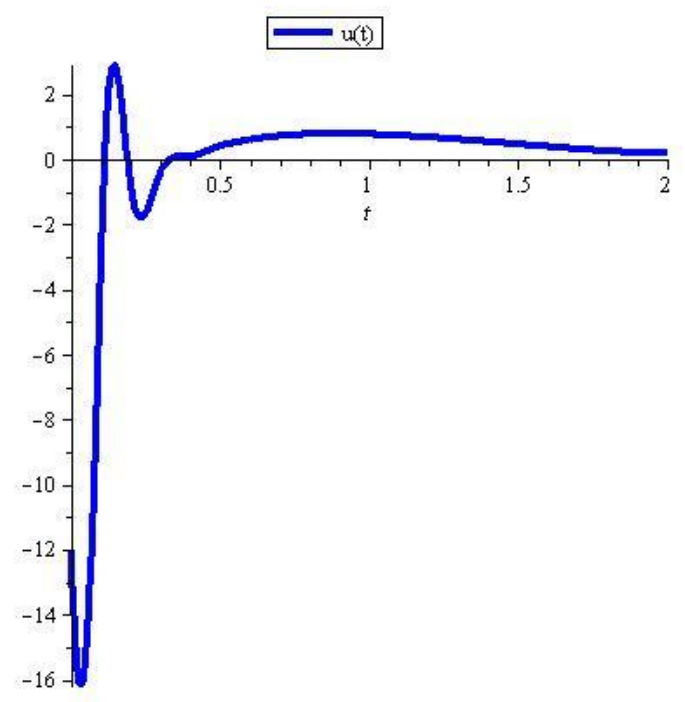

Fig.5 The graph of approximated control

\section{Conclusions}

In this paper, we studied optimal control problems for switched systems in which a pre-specified sequence of 
active subsystems is given. Based on Bezier control points, we proposed a method to obtain the approximated trajectories and control. This note first transcribes an optimal control problem in to an equivalent problem parameterized by the switching instants and then uses the Bezier control points method. The original new problem is converted into a nonlinear programming problem (NLP) by applying Bezier control points method, whereas the MATLAB optimization routine FMINCON is used for solving resulting NLP. Numerical example shows that the proposed method is efficient and very easy to use.

\section{References}

[1] A. Bemporad, F. Borrelli, M. Morari, Optimal controllers for hybrid systems: stability and piecewise linear explicit form. Proceedings - 39th IEEE Conference on Decision Control, 2000, pp. 1810-1815.

[2] J. Lu, L. Liao, A. Nerode, J. H. Taylor, Optimal control of systems with continuous and discrete states. Proceedings - 32nd IEEE Conference on Decision Control, 1993, pp. 2292-2297.

[3] J. Zheng, , T. W. Sederberg, R. W. Johnson, Least squares methods for solving differential equations using Bezier control points. Applied Numerical Mathematics, v48, 2004, pp. 237-252.

[4] H .W. J. Lee, L. S. Jennings, K. L. Teo, V. Rehbock, Control parametrization enhancing technique for time optimal control problems. Dynamic Systems and Applications, v6, 1997, pp. 243-262.

[5] K. Gokbayrak, C. G. Cassandras, Hybrid controllers for hierarchically decomposed systems. Proceedings of the Hybrid Systems: Computation Control (HSCC 2000), 2000, pp. 117-129.

[6] L. Wu, J. Lam, Sliding mode control of switched hybrid systems with time-varying delay. International Journal of Adaptive Control and Signal Processing, v22, 2008, pp. 909-931.

[7] M. S. Branicky, V. S. Borkar, S. K. Mitter, A unified framework for hybrid control: model and optimal control theory. IEEE Transactions on Automatic Control, v43, 1998, pp. 31-45.

[8] A. Giua, C. Seatzu, C. V. D. Mee, Optimal control of autonomous linear systems switched with a preassigned finite sequence. Proceedings of the IEEE International Symposium on Intelligent Control, 2001, pp. 144-149.

[9] B. Farhadinia, K. L. Teo, R. C. Loxton, A computational method for a class of non-standard time optimal control problems involving multiple time horizons. Mathematical and Computer Modelling, v49, 2009, pp. 1682-1691.

[10] B. Lincoln, B. M. Bernhardsson, Efficient pruning of search trees in LQR control of switched linear systems. Proceedings - 39th IEEE Conference on Decision Control, 2000, pp. 1828-1833.
[11] H. H. Mehne, M. H. Farahi, A. V. Kamyad, MILP modelling for the time optimal control problem in the case of multiple targets, Optimal Control Applications and Methods, v27, 2006, pp. 77-91.

[12] J. Johansson, Piecewise linear control systems. Ph.D. dissertation, Lund Inst. Technol, Lund, Sweden, 1999.

[13] M. Egerstedt, Y. Wardi, F. Delmotte, Optimal control of switching times in switched dynamical systems. Proceedings of the IEEE conference on decision and control, v3, 2003, pp. 2138-2143.

[14] X. Xu, P. J. Antsakalis, Optimal control of switched systems based on parameterization of the switching instants. IEEE Transactions on Automatic Control, v 49, n1, 2004, pp. 2-16.

[15] X. Xu, G. Zhai, S. He, Some results on practical stabilizability of discrete-time switched affine systems. Nonlinear Analysis: Hybrid Systems. v4, 2010, pp. 113-121.

[16] B. Niua, J. Zhaoa, Stabilization and L2-gain analysis for a class of cascade switched nonlinear systems: An average dwell-time method. Nonlinear Analysis: Hybrid Systems, v5, 2011, pp. 671-680.

[17] I. Hwang, J. Li, D. Dua, Numerical algorithm for optimal control of a class of hybrid systems: differential transformation based approach. International Journal of Control, v81, n2, 2008, pp. 277-293.

Fateme Ghomanjani is a PHD student in control theory at the Department of Applied Mathematics, Ferdowsi University of Mashhad, Iran. She received her B.S and M.Sc respectively in Applied Mathematics and Operation Research from Ferdowsi University of Mashhad in 2006, 2008. Her scientific interests include optimal control, optimization, delay differential equations, and switched systems.

Mohammad Hadi Farahi is a Full Professor at the Department of Applied Mathematics, School of Mathematics, Ferdowsi University of Mashhad, Iran. He obtained his B.S, M.Sc and PHD, respectively in Ferdowsi University of Mashhad, Iran in 1972, Branel University, UK in 1978, and Leeds University, Uk in 1996. He has published more than 50 technical paper in international journals, and also four textbooks. His scientific interests include optimal control, optimization, sliding mode control, bio-mathematics, ODE's, PDE's, and approximation techniques. 